\title{
An Economic Method for Large-Scale Patterning and Electric Measurement of Nanowires
}

\author{
Mei Liu, ${ }^{1,2}$, Ying Chen ${ }^{2,3}$, Qiuquan $\mathrm{GuO}^{4}$, Ruying $\mathrm{Li}^{2}$, Xueliang Sun², and Jun Yang ${ }^{2,4, *}$ \\ ${ }^{1}$ School of Mechatronics Engineering and Automation, Shanghai University, 200072, China \\ ${ }^{2}$ Department of Mechanical and Materials Engineering, the University of Western Ontario, \\ London, Ontario, Canada, N6A $5 B 9$ \\ ${ }^{3}$ Engineering Research Center of Nano-Geomaterials of Ministry of Education \\ (China University of Geosciences), Wuhan 430074, China \\ ${ }^{4}$ Biomedical Engineering Program, the University of Western Ontario, London, Ontario, Canada, N6A 5B9
}

\begin{abstract}
Assembly and alignment of nanomaterials, such as nanowires or nanotubes, are critical steps for integrating functional nanodevices by the bottom-up strategy. Here, we design, fabricate and characterize an economic microfluidic approach, called asymmetrical hydrodynamic focusing, as a simple way to manipulate and align silver nanowires, and thus test nanowires' electric characteristics easily. With the electrode array pre-defined, the density, position and width of the nanowire arrays on the electrode array can be readily tuned by adjusting the flow duration and flow rates of the sheath flow and the sample flow in the asymmetrical hydrodynamic focusing process. Not only is an easy way to implement electric test on nanowires, this approach also has great potential in mass integration and assembly of large arrays of functional nanodevices.
\end{abstract}

Keywords: Hydrodynamic Focusing, NW Assembly, Surface Treatment, Electric Test.

\section{INTRODUCTION}

Nanostructures, such as nanowires (NWs) and nanotubes (NTs), which have remarkable electronic properties, have been heavily explored as highly promising materials in various applications, such as microelectronics, biological engineering, electrooptical technology, environment engineering and so on. However, the electric properties of NWs need to be tested before putting into applications. Transmission electron microscope (TEM) is a feasible method for electric test on single NWs in situ, however, a more efficient and economic preparatory assembly technique is still required for later large scale test.

A practical method is to pattern a large amount of NWs simultaneously onto electrodes, then test the electric characteristics of the NWs simply by a probe station in air, which is more reliable, more efficient and more cost-effective. By now various approaches have been proposed for aligning NWs in large scale, and then carrying out the electric test. For example, Langmuir-Blodgett (LB) techniques can be applied to assemble high-density NW arrays. ${ }^{1-4}$ In this method, NWs suspended at an airliquid interface can be assembled into a closed-paced

\footnotetext{
*Authors to whom correspondence should be addressed.
}

dense single-layer NW films through compression within an LB trough. Dip coating is used to transfer the singlelayer NW film from the air-liquid interface onto a solid substrate. The density of the deposited NWs is controlled by the speed of withdrawing the substrate from the LB trough and the pressure applied to the assembly within the LB trough. Limitations of this technique include reorganization of the NWs during dip coating that leads to overlapping features and gaps within the dense arrays of NWs. ${ }^{2}$ Photolithography was applied on the transferred NW film to remove unwanted areas and lay the testing electric circuit. With all the NWs orienting to the same direction with an appropriate density, the chances that electrodes lay over NWs are greatly increased.

The relative mechanical movement of two solid surfaces against each other can also generate a shear force, which can direct the assembly of vertically grown NWs into arrays with an orientation parallel to the direction of the applied force.$^{5-7}$ But the density of the NWs was hard to control. Assembly of NWs can also be driven by electrostatic interactions. ${ }^{8-13}$ These interactions rely on either the inherent polarizability of the nanostructure or a surface modification of this nanostructure to adopt a specific charge. Confining the fluid flow to a microfluidic channel can also establish a uniform shear drag force that can 
reorient the direction the flowing NWs. ${ }^{14-16}$ These NWs adhere to the surface and adopt an orientation parallel to the fluid flow. The density of NWs can be controlled by changing the concentration of NWs within the suspended liquid and flow time. In addition, the alignment can also be driven by intermolecular forces, ${ }^{17-19}$ electric fields ${ }^{20-29}$ or magnetic fields, ${ }^{30-34}$ but those methods set limits on their applicable NW material.

So here an asymmetrical hydrodynamic-focusing NW aligning method was proposed, which is an extension of our previous work. ${ }^{35-36}$ Using this approach, we aligned a large amount of NWs over gold electrodes controllably, by defining the average distance between NWs nearly equal to that of the electrode width, resulting in multiple NWs bridging corresponding electrode pairs, so the electric characteristics of the NWs can be easily tested. This approach would have great potential in massive production/fabrication of nanoelectronic, nanooptoelectronic and nanoelectromechanical systems.

\section{CONCEPTS AND PRINCIPLES}

\subsection{Principles of 2D Hydrodynamic Focusing}

Hydrodynamic focusing is a common phenomenon of flow dynamics, which is applied to assemble NWs onto gold electrodes in a controllable manner in this study. The smooth profile of the convergent part was show in Figures 1(a) and (b) as adopted from Lee's paper. ${ }^{37}$ Also, in the device design, the inner nozzle is put at the middle of the outer nozzle. ${ }^{37-38}$ The sample flow (supplied from the inlet channel) is constrained laterally at a specified position in the microchannel by two neighbouring sheath flows from the side channels. The flow inside the microfluidic channel is considered to be laminar, and the diffusion and mixing between the focused stream and sheath flows are assumed negligible. Thus, the sample flow and the sheath flows will form a stable two-phase flow configuration, with the sheath flows enveloping the sample flow in a straight line. The width of the focused stream could be controlled through adjusting the flow rates of the sheath flows and the sample flow: when the flow ratio $R$, defined as the ratio of sheath flow over sample flow is low, the sample stream will broaden out; while if the flow ratio $R$ is high, the focused stream will narrow down. The position of the focused stream could be controlled through adjusting the flow rates of the two sheath flows: as the flow rate of one sheath flow increases over the other one, the focused stream will be pushed away from the increasing sheath flow to the decreasing sheath flow. Detailed calculation and theoretical predictions of hydrodynamic focusing can be found in Lee's paper. ${ }^{37}$

\subsection{Principles of NW Assembly}

In this work, both symmetrical and asymmetrical hydrodynamic flow focusing was employed, where the flow rates from the two sheath flows and one sample flow were adjusted for controllable NW assembly. NWs assembly was accomplished by injecting a NW solution $\left(Q_{i}\right.$ in Fig. 1(a)), inside the sheath flows $\left(Q_{\mathrm{s} 1}\right.$ and $Q_{\mathrm{s} 2}$ in Fig. 1(a)) which was free of NWs. The substrate was chemically treated so the NWs in the sample flow tend to reside on it. The NWs was kept in the sample flow direction as immobilizing on the substrate (shown in Fig. 1(c)). After the flow process, only the focused-stream covered area will be covered with well-aligned NWs. By tuning the flow rates of the sheath flows and sample flow, the width and position of NW distribution can be readily manipulated, while the density of NW deposition could be controlled by adjusting the NW concentration and flow duration of the sample flow.

\subsection{Electric Characterization of the NWs}

As most NWs are aligned in the flow direction with predefined position and density over the electrode array, the NWs will be more apt to residue on the electrode pattern, resulting in NWs connecting electrode pairs, as shown in Figure 1(c). With optimized flow protocols, considerable amount of electrode pairs will be bridged by NWs, whose electric characteristics can then be tested directly.

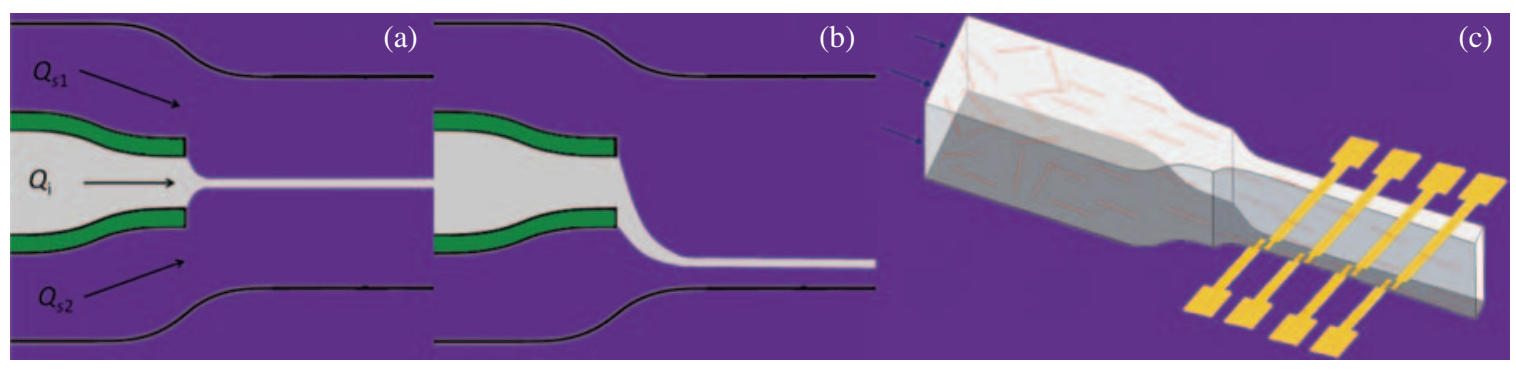

Fig. 1. The schematics of asymmetrical hydrodynamic focusing and NW assembly on gold electrodes. (a) Top view of the symmetrical hydrodynamic focusing process. $Q_{i}$ is the volumetric sample flow rate; $Q_{\mathrm{s} 1}$ and $Q_{\mathrm{s} 2}$ are the volumetric flow rate of two sheath flows. (b) Top view of the asymmetrical hydrodynamic focusing process. (c) Side view of the focused stream showing NWs flowing in the sample stream and depositing on the device substrate carrying gold electrodes. 


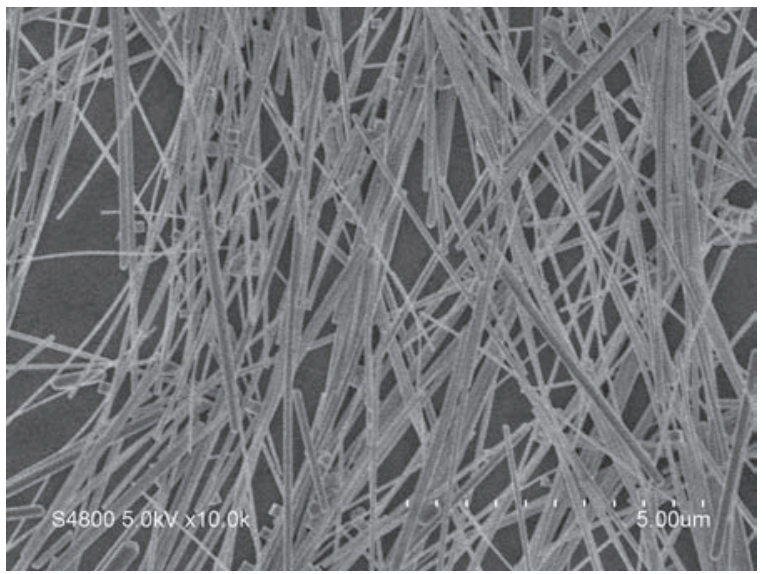

Fig. 2. The SEM image of synthesized AgNWs by the solution-phase method.

In a conducting circuit including a probe station, a gold electrode substrate and the NW, the whole electric resistance in the circuit is composed of four parts:

$$
R_{\text {total }}=R_{\mathrm{p} 1}+R_{\mathrm{p} 2}+R_{\mathrm{p} 3}+R_{\mathrm{p} 4}
$$

Each item on right side of Eq. (1) represents part of the resistance in the conducting circuit, where $R_{\mathrm{p} 1}$ is the contact resistance between the test probes and gold contact pads, $R_{\mathrm{p} 2}$ is the resistance of the gold wires and the probe station itself, $R_{\mathrm{p} 3}$ is the contact resistance between the electrodes and the NW, and $R_{\mathrm{p} 4}$ is the resistance of the NW.

The contact resistance $R_{\mathrm{p} 3}$ and resistance of the NW $R_{\mathrm{p} 4}$ follow the equations:

$$
\begin{aligned}
R_{\mathrm{p} 3} & =\frac{\rho_{\text {Contact }}}{\pi L_{1} r} \\
R_{\mathrm{p} 4} & =\rho_{\mathrm{NW}} \frac{L_{2}}{\pi r^{2}}
\end{aligned}
$$

Where $\rho_{\text {contact }}$ is the contact resistivity between the electrodes and the $\mathrm{NW}, \rho_{\mathrm{NW}}$ is the resistivity of the $\mathrm{NW}, r$ is the radius of the corresponding $\mathrm{NW}, L_{1}$ is the contact length between the NW and the electrodes, $L_{2}$ is the working length of the NW in the circuit. Eqs. (1)-(3) can be combined together to derive $\rho_{\text {contact }}$ and $\rho_{\mathrm{NW}}$.

\section{EXPERIMENTAL PROCESSES}

To testify the feasibility of this symmetrical/asymmetrical hydrodynamic focusing approach, a symmetrical microfluidic device was designed and fabricated and NW flow process was demonstrated to assemble NW asymmetrically on the electrode pattern. Detailed experimental process will be discussed, including AgNWs synthesis, microfabrication process of the electrode substrate and microfluidic device, functionalizing substrate, the hydrodynamic flow process, and the electric test process.

\subsection{AgNWs Synthesis}

The development of integrated circuits (IC) requires interconnects of low resistivity, high electromigration resistance and high ability to carry a large current density. Silver is promising for the next generation of interconnects due to its highest conductivity and high electromigration resistance at ambient conditions. AgNWs are also a very good model system for studying the proposed process since they can be synthesized in a relatively high yield and uniformity. In addition, the electrical properties of AgNWs were seldom explored.

A typical solution-based synthesis procedure was implemented for AgNWs, by reducing $\mathrm{AgNO}_{3}$ with EG (ethylene glycol, Sigma Aldrich) in the presence of PVP (Polyvinylpyrrolidone, Sigma Aldrich), which was injected into refluxed EG. Figure 2 shows the synthesized AgNWs with diameters around $80 \mathrm{~nm}$ and lengths of about $15 \mu \mathrm{m}$.

\subsection{Fabrication of the Substrate}

This substrate design was similar to that of a published paper by Lieber's group. ${ }^{39}$ As shown in Figure 3 in our previously published paper, ${ }^{35}$ the whole electric circuit is symmetrically and densely designed, and there are totally

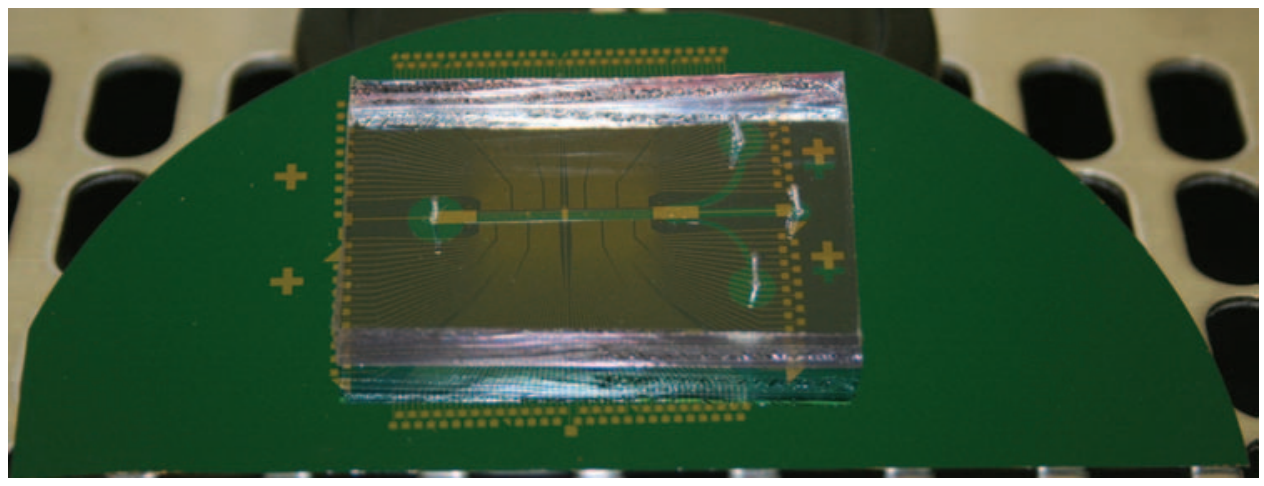

Fig. 3. The hydrodynamic focusing microfluidic device. 
ten groups of electrode-pair collections, while each collection has eighteen pairs of electrodes. In each collection, one line is the common line, shared by eighteen electrodes. The common lines and the corresponding lines are extended outward to gold pads, which can be easily accessed by testing probes of a probe station. For the convenience of distinguishing the lines, the common lines are connected to triangular contact pads, while their corresponding lines are connected to rectangular contact pads. The markers are for the alignment between the electric circuit wafer and PDMS mold.

The electrode pattern on the substrate was prepared by a typical lift-off photolithography process. In detail, the substrate was first coated with two layers of photoresists separately: LOR5 and Shipley1805 (MicroChem, MA). LOR5 was used to control undercut. The electrode pattern was transferred to the substrate by photolithography. After the substrate was developed, Cr (100 ̊) and Au (1000 $)$ thin films were evaporated on the substrate sequentially. The photoresists were then dissolved by Remover PG at $60{ }^{\circ} \mathrm{C}$ in an ultrasonic vibrator. Thus, electrodes were patterned on the substrate. The substrate was then cleaned with isopropyl alcohol (IPA), deionised (DI) water and dried with $\mathrm{N}_{2}$.

\subsection{Microfluidic Device Fabrication}

Firstly the master mold structure of $25 \mu \mathrm{m}$ high SU-8 photoresist was fabricated by photolithography on a silicon wafer. The wafer was pre-coated with hexamethyldisiloxane (HMDS) to facilitate subsequent peeling off of the PDMS layer.

A mixture of PDMS prepolymer and curing agent (Sylgard 184, Dow Corning) was poured over the positive master mold. After curing, a negative relief made of PDMS was peeled off. Holes were punched at the center of the reservoir area and the relief was sealed to the previously prepared substrate to form a closed channel. The electrodes area must be inside the microchannel. The device was connected to syringe pumps and a waste beaker by plastic tubing. Figure 3 shows the fabricated symmetrical device.

\subsection{Device Treatment Process with APTES}

The substrate surface in the channel was cleaned with pure ethanol first and then functionalized with an $\mathrm{NH}_{2}-$ terminated self-assembled monolayer (SAM) by pumping in the device channel with a $1 \mathrm{mM}$ alcohol solution of 3aminopropyltriethoxysilane (APTES, Sigma Aldrich) and staying for $10 \mathrm{~h}$. After $10 \mathrm{~h}$, the device was heated at $110{ }^{\circ} \mathrm{C}$ for $10 \mathrm{~min} .{ }^{14}$ After this treatment, the channel surface will be turned hydrophilic. To keep the channel surface hydrophilic, water should be kept flowing through the system before use.

\subsection{The NW Assembly Process}

The ethanol solution with suspended AgNWs was injected from the center channel and focused hydrodynamically into a narrow stream constrained by DI water from the sheath channels. The width and position of the focused sample flow can be readily tuned by adjusting the flow rates of the sheath flows and sample flow, controlled with three external pumps. To obtain a steady distribution of AgNWs on the substrate, the velocity of the focused sample was maintained around $3 \mathrm{~mm} / \mathrm{s}$.

Three specific protocols, as listed in Table I, were developed based on numbers of systematic experiments on difference devices to study the hydrodynamic focusing flow behaviour and the AgNW assembly process. The focusing processes are respectively presented in Figures 4(a)-(c). A stable focused sample stream could be formed while the appropriate ratio between the sheath flow rate and the sample flow rate was applied. As the sheath flows were tuned, the focused stream was shifted across the microchannel, resulting in AgNWs depositing on selected lanes, as shown in Figure 5, where the three AgNWs lanes were the corresponding results of the three protocols in Figure 4.

After set flow duration of each protocol, the PDMS replica of the device was removed from the substrates and NW patterns were imaged by SEM or an optical microscope. And the electric characteristics were tested with a probe station.

Flow protocols with longer flow duration was also implemented to test the effect of flow time, as shown in Figures 6(a) and (b).

\subsection{Electric Test of AgNWs}

For the AgNWs to be tested, the metal contact pads and interconnection lines should be well-formed, continuous and sharp with a good yield of single AgNW spanning two electrodes. All electrical measurements were carried out in air at room temperature. Firstly, tweezers were used to clamp the edge of the substrate chip and hold it firmly. Secondly, for air characterization, individual $\mathrm{AgNW}$ devices on the microfluidic substrate were connected to the measurement electronics by the probe station. Probes were brought into contact with the outer metal contact pads on the substrate, while being viewed optically.

Table I. Flow protocols for AgNW alignment.

\begin{tabular}{lccccccc}
\hline $\begin{array}{l}\text { Flow } \\
\text { protocol }\end{array}$ & $\begin{array}{c}\text { Sheath } \\
\text { flow } Q_{\mathrm{s} 1} \\
(\mathrm{~mL} / \mathrm{h})\end{array}$ & $\begin{array}{c}\text { Sheath } \\
\text { flow } Q_{\mathrm{s} 2} \\
(\mathrm{~mL} / \mathrm{h})\end{array}$ & $\begin{array}{c}\text { Sample } \\
\text { flow } Q_{i} \\
(\mathrm{~mL} / \mathrm{h})\end{array}$ & $\begin{array}{c}\text { Flow } \\
\text { rate } \\
\text { ratio } R\end{array}$ & $\begin{array}{c}\text { Focused } \\
\text { width } \\
(\mu \mathrm{m})\end{array}$ & $R^{\prime}$ & $\begin{array}{c}\text { Flow } \\
\text { duration } \\
(\mathrm{min})\end{array}$ \\
\hline 1 & 0.03 & 0.17 & 0.03 & 6.67 & 120 & 0.8 & 20 \\
2 & 0.17 & 0.03 & 0.03 & 6.67 & 120 & 0.2 & 20 \\
3 & 0.12 & 0.12 & 0.03 & 8 & 100 & 0.45 & 20 \\
\hline
\end{tabular}

Note: $R^{\prime}$ is defined as the proportion that sheath flow 1 occupies in the outlet channel. 


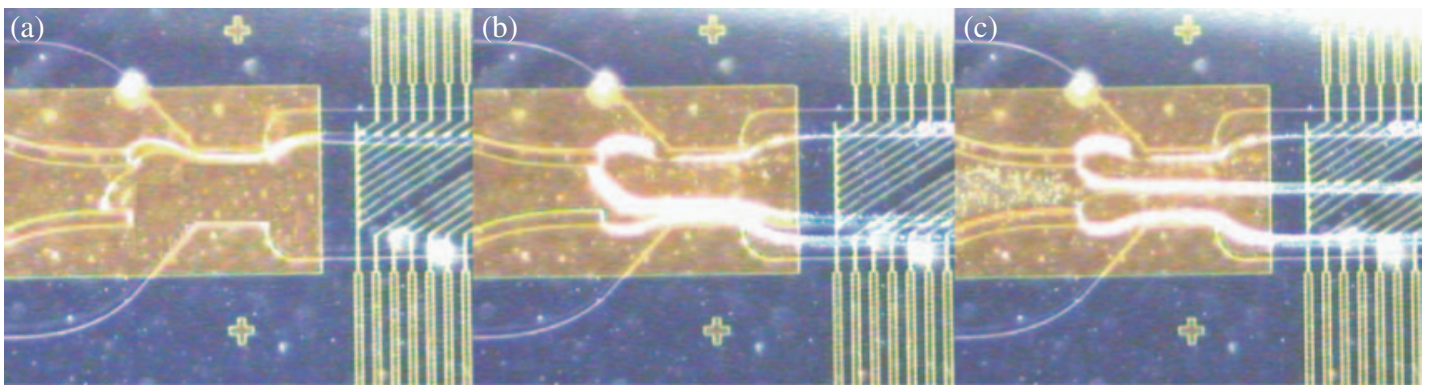

Fig. 4. The asymmetrical hydrodynamic focusing process. (a) Flow Protocol 1; (b) Flow Protocol 2; (c) Flow Protocol 3.

The current-voltage characteristics of AgNW were measured using a Keithley 6517A electrometer with the probe station at room temperature. The $I-V$ curves in Figure 7(a) were obtained by sweeping up and down a DC voltage, ranging from -0.05 to $0.05 \mathrm{~V}$, applied between electrodes pairs as indicated in Figure 7(b).

\section{RESULTS AND DISCUSSION}

\subsection{The Hydrodynamic Focusing Process}

$100 \%$ alcohol with dispersed AgNWs was used as the sample flow because AgNWs are better dispersed in alcohol than in water.
The width of the focused stream can be controlled by the flow ratio of the sheath flow over the sample flow $R$. As the ratio $R$ increases, the width of the focused sample stream would be reduced accordingly. And as one sheath flow rate increases, it will occupy more space in the outlet microchannel, where three flow configurations at steady state are shown respectively corresponding to the three flow protocols listed in Table I in Figure 4. As shown in the figures, both the sample and sheath flows were transparent, and distinct borderlines were located among them. The sample flow from the inlet channel was firstly narrowed down by the sheath flows, and its width remains constant in the downstream of the channel. As the sample fluid flowed in the channel, the AgNWs inside

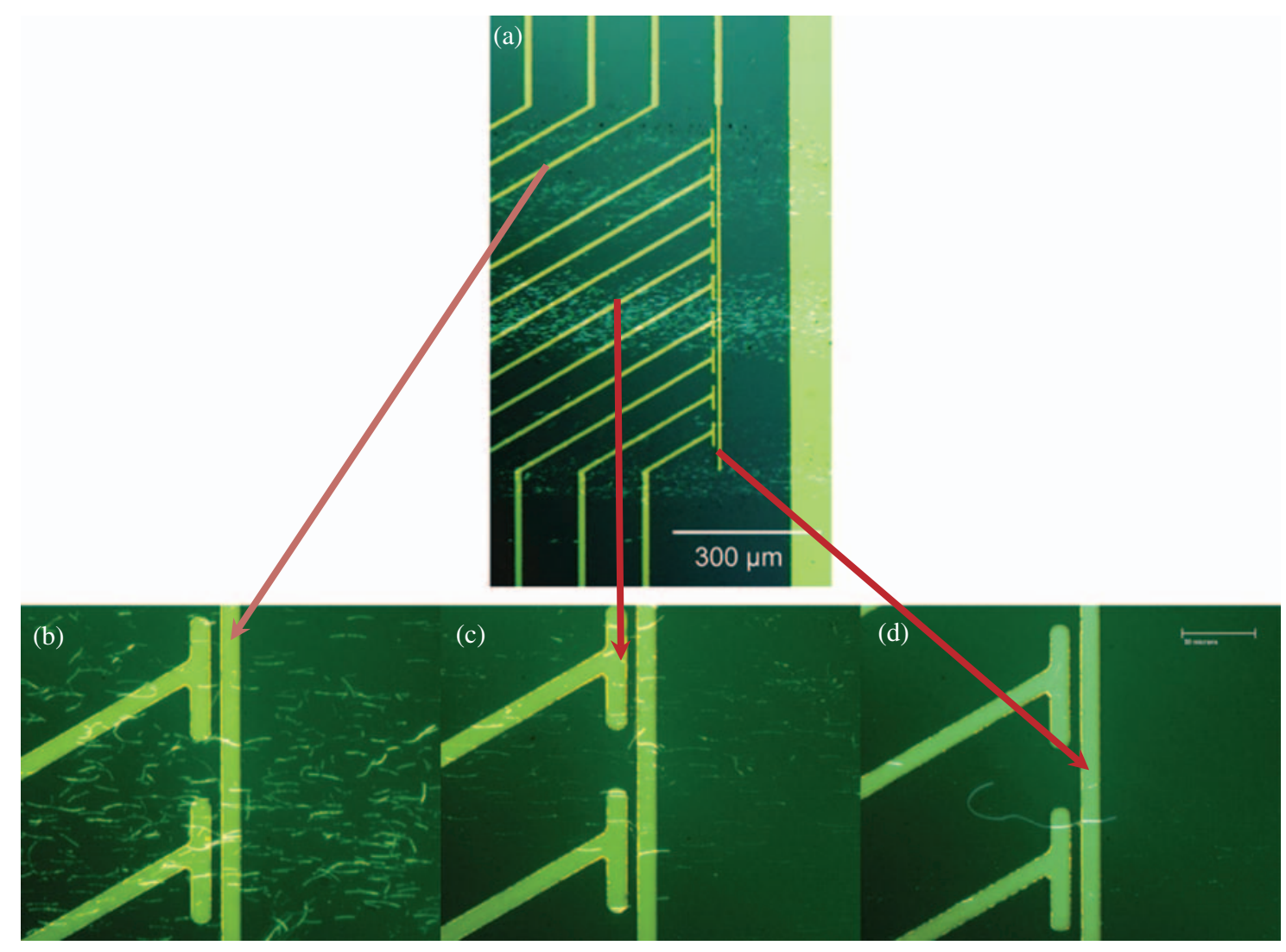

Fig. 5. Electrodes covered with three lanes of AgNWs. (a)-(c) are respectively the three lanes on different electrodes. 

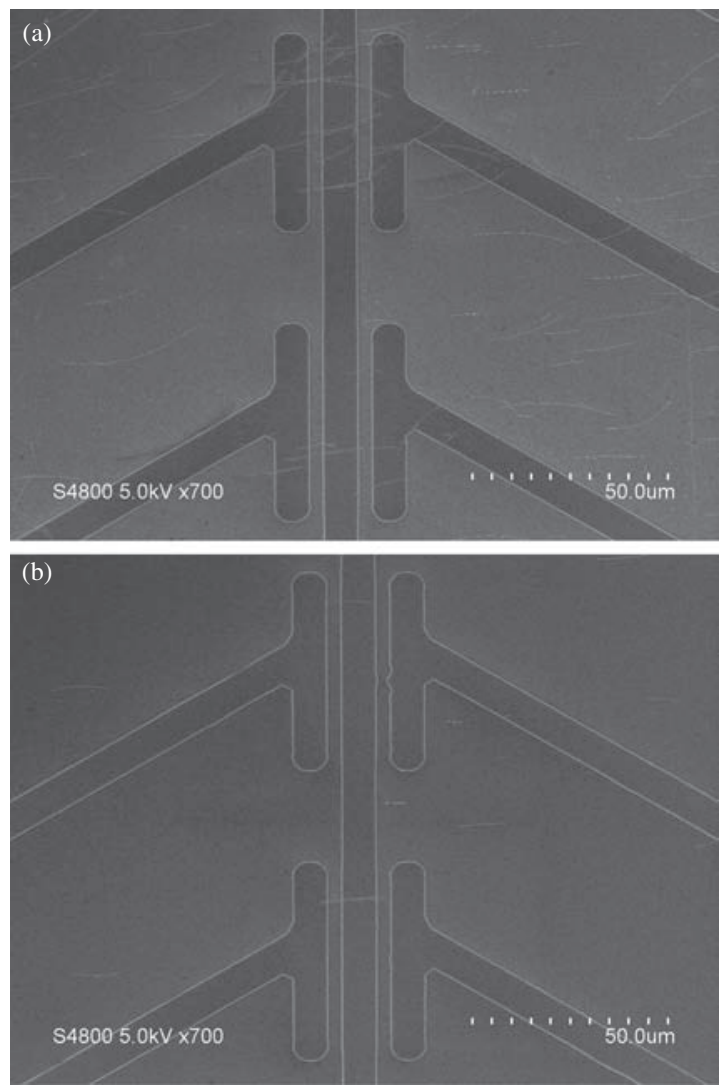

Fig. 6. Electrodes covered with AgNWs. (a) High density AgNWs on the substrate; (b) Low density AgNWs on the substrate.

would deposit on the substrate underneath and some will deposit on the electrodes randomly, since some electrodes are immersed in the sample region. Since three different protocols were used in the experiment, three discrete lanes of AgNWs will deposit on the electrode arrays, as shown in Figure 5. Compared with our previous symmetrical hydrodynamic focusing work, ${ }^{35}$ the asymmetrical process exhibited more control on the flow, where not only the width, but also the location of the NW array can be easily adjusted.

The aligned NW array readily extends over hundreds of micrometers. Indeed, alignment of the NWs has been found to extend up to millimeter length scales and is limited by the size of the fluidic channels only. Therefore this method is really robust to form size-flexible NW patterns.

\subsection{AgNW Assembly Characterization}

Notably, images in Figure 5 show clearly that there are three lanes of AgNWs arrays on the electrode substrate, resulting in AgNWs selectively connecting electrodes.

Figures 5 and 6(a) shows that multiple AgNWs were connected with electrodes, while Figure 6(b), experimental results of shorter depositing time shows that individual NWs are connected with electrodes with about $50 \mu \mathrm{m}$ pitches. There are also NWs that fall between the electrodes, as expected statistically, although these are not
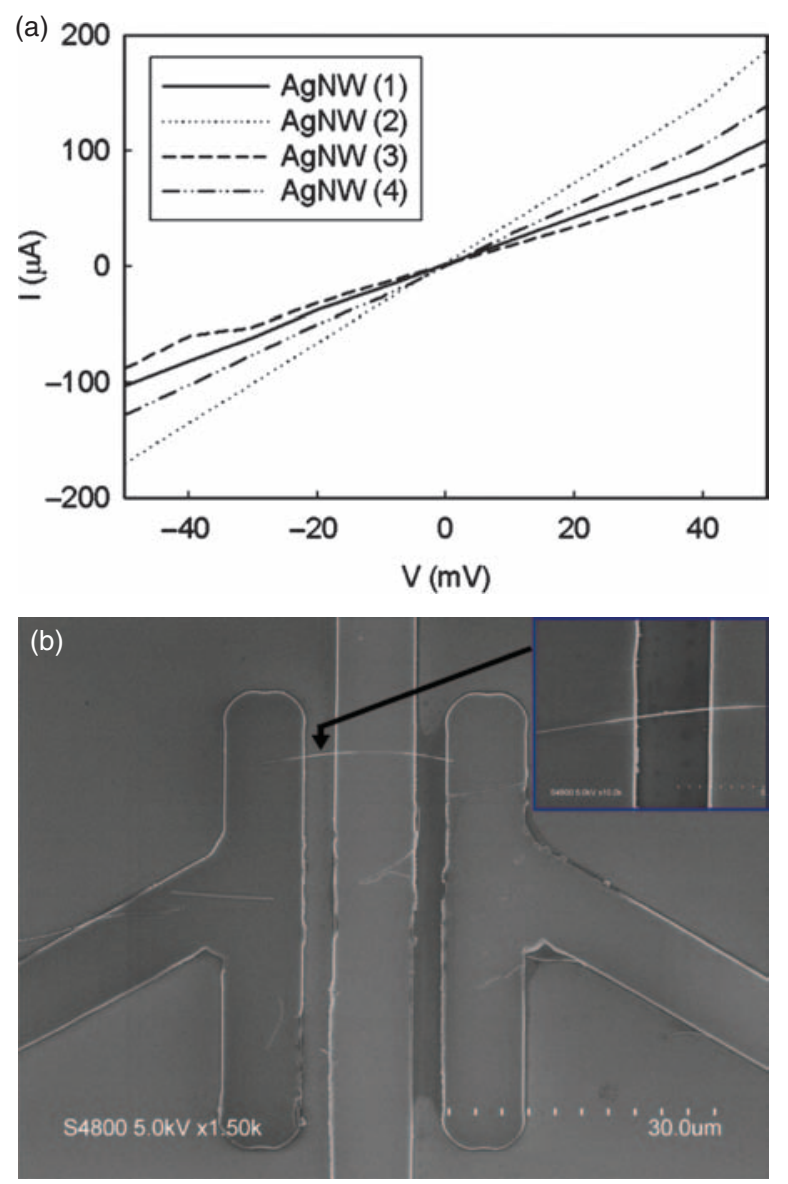

Fig. 7. (a) The $I-V$ curve of four example AgNWs. (b) The corresponding SEM image of a tested AgNW. The inset in is the enlarged image of the AgNW.

expected to affect the interconnected active devices. The average NW spacing can be reduced further to increase the connection yield, although this also increases the probability of having multiple NWs connected to one electrode, as shown in Figure 6(a).

Typical examples of assembled NW arrays (Figures 5 and 6) show that virtually all the NWs were aligned along the flow direction, though there were also some small deviations with respect to the flow direction, which were caused by instabilities during the flowing process. With optimal experimental parameters and conditions, this method can obtain large-scale assembled AgNW arrays.

There are several factors controlling the alignment and average separation of the NWs:

(1) the flow rate. With increasing flow rates, the deviation of the NWs' alignment with respect to the flow direction substantially becomes narrower. Higher flow rates produce larger shear forces and hence lead to better NW alignment. (2) The NW deposition coverage can be controlled by the flow duration. Experiments carried out at given operating conditions showed that the NW density increases systematically with increase of the flow duration. Figures 6(a) and (b) showed the well assembled and 
aligned AgNWs along the flow direction, as expected, with longer flow time, deposited AgNWs will be denser, as shown in Figure 6(a), multiple AgNWs are deposited on the one pair of electrode. With flow time shorter, less AgNWs will deposit on the substrate, as shown in Figure 6(b), only one single AgNW is lying on one pair of electrode.

(3) The deposition rate and hence the average NW separation distance versus time also depend strongly on the surface chemical functionality.

(4) NW concentration in the solution. With higher density of NWs, the assembled AgNW arrays become denser. Those features were also demonstrated in our early-stage work in Ref. [36].

\subsection{Electric Property of AgNWs}

To test the electric property of AgNWs, most common problems arise from poor electrical contacts between the electrodes and AgNWs. So much attention should be paid to cleaning immediately before $\mathrm{AgNWs}$ deposition.

The electrical characterizations of four randomly chosen functional NW devices within the large arrays (Fig. 7) show linear $I-V$ curves as expected, which means, the electric contacts between the electrode and AgNW are good and ohmic at the junction, and the AgNWs exhibits electric property of metal material. The voltage applied was quite low $(0.05 \mathrm{~V})$, ensuring that the $\mathrm{AgNW}$ tested will not be destroyed due to overcurrent.

From the curves it was obtained that the whole electric resistance in the four circuits are respectively $288 \Omega$, $324 \Omega, 546 \Omega, 603 \Omega . R_{\mathrm{p} 1}$ and $R_{\mathrm{p} 2}$ can be measured directly with the probe station by short-passing the electrode pairs. Using Eqs. (1)-(3), finally $R_{\mathrm{p} 4}$ were calculated as in the range of $60-80 \Omega$, and the electric resistivity of the synthesized AgNWs in the range of 19.5-25.2 $\mu \Omega \cdot \mathrm{cm}$, which is consistent with other groups' results. ${ }^{40-42}$ The contact resistivity between the AgNWs and the electrodes is calculated as in the range of $0.33-0.43 \mu \Omega \cdot \mathrm{cm}^{2}$.

The deviation in those obtained total resistance were results of floating $R_{\mathrm{p} 1}, R_{\mathrm{p} 2}, R_{\mathrm{p} 3}$ and $R_{\mathrm{p} 4}$. To improve the experimental outcome, $R_{\mathrm{p} 1}$ could be eliminated by implementing a four-point-probe measurement method. $R_{\mathrm{p} 3}$ could be greatly reduced by carrying out annealing on the substrate after AgNWs deposition to enhance the electric contact between the gold electrode and AgNWs.

In this paper millimeterscale arrays containing hundreds of AgNWs-electrode pairs fabricated by tuneable hydrodynamic focusing were shown to exhibit high performance with high reproducibility and scalability from $\mu \mathrm{m}$ to $\mathrm{cm}$ level. This basic approach is scalable and thus can be used to produce devices in the nanometer size regime over large areas and on selected positions. Because the average NW spacing can be controlled down to the nanometer scale by adjusting the duration time and sample concentration, a much smaller device size and a higher density of device integration can be readily achieved when existing high-resolution parallel lithography techniques.

The general applicability of this approach to a wide range of NW materials and organized NW structures could also enable a pathway to highly functional and integrated nanosystems.

\section{CONCLUSION}

Successful synthesis of high-quality AgNWs and its high degree of alignment by asymmetrical hydrodynamic focusing with controlled density and large area coverage will open up possibilities of utilizing them for nanoelectronics applications. To illustrate this potential, arrays of independently addressable electric circuits were photolithographically patterned on Si substrates with AgNWs positioned and aligned by asymmetrical hydrodynamic focusing for electric testing. The proposed method provides a convenient control on the width, density and position of assembled NW arrays by just simply adjusting the flow rates of the sheath and sample flows in the asymmetrical hydrodynamic focusing process. Among the patterning methods developed so far, asymmetrical hydrodynamic focusing is controllable and tuneable, which is unique in all the NW assembly methods.

Acknowledgments: J. Yang is grateful for the financial support from Natural Science and Engineering Research Council of Canada (NSERC) and The Canada Foundation for Innovation (CFI). The authors would like to also acknowledge the nanofabrication laboratory of the University of Western Ontario for the use of their laboratory facilities.

\section{References and Notes}

1. S. Acharya, A. B. Panda, N. Belman, S. Efrima, and Y. Golan, $A d v$. Mater. 18, 210 (2006).

2. J. Park, G. Shin, and J. S. Ha, Nanotechnology 19, 395303 (2008).

3. A. Tao, F. Kim, C. Hess, J. Goldberger, R. R. He, Y. G. Sun, Y. N. Xia, and P. D. Yang, Nano Lett. 3, 1229 (2003).

4. D. W. Wang, Y. L. Chang, Z. Liu, and H. J. Dai, J. Am. Chem. Soc. 127, 11871 (2005).

5. Z. Y. Fan, J. C. Ho, Z. A. Jacobson, R. Yerushalmi, R. L. Alley, and H. Razavi, Nano Lett. 8, 20 (2008)

6. A. Javey, S. Nam, R. S. Friedman, H. Yan, and C. M. Lieber, Nano Lett. 7, 773 (2007).

7. R. Yerushalmi, Z. A. Jacobson, J. C. Ho, Z. Fan, and A. Javey, Appl. Phys. Lett. 91, 203104 (2007).

8. K. Heo, E. Cho, J. E. Yang, M. H. Kim, M. Lee, B. Y. Lee, S. G. Kwon, M. S. Lee, M. H. Jo, H. J. Choi, T. Hyeon, and S. Hong, Nano Lett. 8, 4523 (2008).

9. J. Kang, S. Myung, B. Kim, D. Oh, G. T. Kim, and S. Hong, Nanotechnology 19, 095303 (2008).

10. Y. K. Kim, S. J. Park, J. P. Koo, G. T. Kim, S. Hong, and J. S. Ha, Nanotechnology 18, 015304 (2007).

11. S. Myung, K. Heo, M. Lee, Y. H. Choi, S. H. Hong, and S. Hong, Nanotechnology 18, 205304 (2007). 
12. S. Myung, J. Im, L. Huang, S. G. Rao, T. Kim, D. J. Lee, and S. H. Hong, J. Phys. Chem. B 110, 10217 (2006).

13. S. Myung, M. Lee, G. T. Kim, J. S. Ha, and S. Hong, Adv. Mater. 17, 2361 (2005).

14. Y. Huang, X. F. Duan, Q. Q. Wei, and C. M. Lieber, Science 291, 630 (2001).

15. M. C. McAlpine, R. S. Friedman, S. Jin, K. H. Lin, W. U. Wang, and C. M. Lieber, Nano Lett. 3, 1531 (2003).

16. M. C. McAlpine, R. S. Friedman, and D. M. Lieber, Nano Lett. 3, 443 (2003).

17. J. K. N. Mbindyo, B. D. Reiss, B. R. Martin, C. D. Keating, M. J. Natan, and T. E. Mallouk, Adv. Mater. 13, 249 (2001).

18. J. Lee, A. A. Wang, Y. Rheem, B. Yoo, A. Mulchandani, W. Chen, and N. V. Myung, Electroanal. 19, 2287 (2007).

19. M. Chen, L. Guo, R. Ravi, and P. C. Searson, J. Phys. Chem. B 110, 211 (2006).

20. T. H. Kim, S. Y. Lee, H. G. Kim, S. H. Kim, C. H. Hong, Y. B. Hahn, and S. K. Lee, J. Nanosci. Nanotechnol. 8, 268 (2008).

21. J. W. Lee, K. J. Moon, M. H. Ham, and J. M. Myoung, Solid State Commun. 148, 194 (2008).

22. M. W. Li, R. B. Bhiladvala, T. J. Morrow, J. A. Sioss, K. K. Lew, J. M. Redwing, C. D. Keating, and T. S. Mayer, Nat. Nanotechnol. 3, 88 (2008).

23. Y. L. Liu, J. H. Chung, W. K. Liu, and R. S. Ruoff, J. Phys. Chem. B 110, 14098 (2006).

24. M. S. Marcus, L. Shang, B. Li, J. A. Streifer, J. D. Beck, E. Perkins, M. A. Eriksson, and R. J. Hamers, Small 3, 1610 (2007).

25. A. Motayed, M. Q. He, A. V. Davydov, J. Melngailis, and S. N. Mohammad, J. Appl. Phys. 100, 114310 (2006).

26. W. R. Small and V. N. Paunov, J. Mater. Chem. 18, 2082 (2008).

27. P. A. Smith, C. D. Nordquist, T. N. Jackson, T. S. Mayer, B. R. Martin, J. Mbindyo, and T. E. Mallouk, Appl. Phys. Lett. 77, 1399 (2000).
28. D. Q. Wang, R. Zhu, Z. Y. Zhou, and X. Y. Ye, Appl. Phys. Lett. 90, 103110 (2007).

29. R. H. Zhou, H. C. Chang, V. Protasenko, M. Kuno, A. K. Singh, D. Jena, and H. Xing, J. Appl. Phys. 101, 073704 (2007).

30. C. M. Hangarter and N. V. Myung, Chem. Mater. 17, 1320 (2005).

31. C. M. Hangarter, Y. Rheem, B. Yoo, E. H. Yang, and N. V. Myung, Nanotechnology 18, 205305 (2007).

32. M. Liu, J. Lagdani, H. Imrane, C. Pettiford, J. Lou, S. Yoon, V. G. Harris, C. Vittoria, and N. X. Sun, Appl. Phys. Lett. 90, 103105 (2007).

33. C. Ooi and B. B. Yellen, Langmuir 24, 8514 (2008).

34. B. Y. Yoo, Y. W. Rheem, W. P. Beyermann, and N. V. Myung, Nanotechnology 17, 2512 (2006).

35. M. Liu, Y. Peng, Q. Guo, J. Luo, and J. Yang, Large-scale patterning and electric test of silver nanowires by microfluidic hydrodynamic focusing, 2010 International Conference on Advances in Materials and Manufacturing Processes, Shenzhen, China (2010).

36. M. Liu, Y. Chen, Q. Guo, R Li, X. Sun, and J. Yang, Nanotechnology 22, 125302 (2011).

37. G. B. Lee, C. C. Chang, S. B. Huang, and R. J. Yang, J. Micromech. Microeng. 16, 1024 (2006).

38. C. I. Hung, B. J. Ke, G. R. Huang, B. H. Hwei, H. F. Lai, and G. B. Lee, J. Fluid Eng.-T. Asme. 123, 672 (2001).

39. F. Patolsky, G. F. Zheng, and C. M. Lieber, Nat. Protoc. 1, 1711 (2006).

40. D. Duffy, J. McDonald, O. Schueller, and G. Whitesides, Anal. Chem. 70, 4974 (1998).

41. J. Liu, Y. Fu, A. Guo, C. Wang, R. Huang, and X. Zhang, Fabrication of silver nanowires in situ in Si chip based on a novel electrochemical method, Proceedings of the 2nd IEEE International Nanoelectronics Conference (INEC 2008), Shanghai, China, March.

42. X. Liu, J. Zhu, C. Jin, L. Peng, D. Tang, and H. Cheng, Nanotechnology 19, 085711 (2008).

Received: $\mathrm{xx}$ Xxxx xxxx. Accepted: $\mathrm{xx}$ Xxxx xxxx. 